\title{
Preoperative fibrinogen / albumin ratio (FAR) Can be used to predict the prognosis of urothelial carcinoma patients after radical cystectomy
}

\section{Lei Liang}

Department of Urology, Peking University First Hospital, Institute of Urology, Peking University, National Urological Cancer Center, Beijing 100034, China

\section{Haifeng Song}

Department of Urology, Beijing Tsinghua Changgung Hospital, Beijing,102200, China

\section{Zhenhua Liu}

Department of Urology, State Key Laboratory of Oncology in South China, Collaborative Innovation Center for Cancer Medicine, Sun Yat-sen University Cancer Center, Guangzhou 510060, P. R. China.

\section{Binglei Ma}

Department of Urology, Peking University First Hospital, Institute of Urology, Peking University, National Urological Cancer Center, Beijing 100034, China

\section{Zhenan Zhang}

Department of Urology, Peking University First Hospital, Institute of Urology, Peking University, National Urological Cancer Center, Beijing 100034, China

\section{Qinhan Li}

Department of Urology, Peking University First Hospital, Institute of Urology, Peking University, National Urological Cancer Center, Beijing 100034, China

Jun Liu

Department of Urology, Peking University First Hospital, Institute of Urology, Peking University, National Urological Cancer Center, Beijing 100034, China

\section{Quan Zhang}

Department of Urology, Peking University First Hospital, Institute of Urology, Peking University, National Urological Cancer Center, Beijing 100034, China

\section{Shiyu Zhu}

Department of Urology, Peking University First Hospital, Institute of Urology, Peking University, National Urological Cancer Center, Beijing 100034, China

\section{Yisen Meng}

Department of Urology, Peking University First Hospital, Institute of Urology, Peking University, National Urological Cancer Center, Beijing 100034, China

Qian Zhang ( $\nabla$ zhangqianbjmu@126.com ) 
Department of Urology, Peking University First Hospital, Institute of Urology, Peking University, National Urological Cancer Center, Beijing 100034, China

\section{Research Article}

Keywords: Bladder cancer, radical cystectomy, fibrinogen / albumin ratio (FAR), prognostic independent risk factors, survival

Posted Date: March 2nd, 2021

DOl: https://doi.org/10.21203/rs.3.rs-256124/v1

License: (c) (7) This work is licensed under a Creative Commons Attribution 4.0 International License. Read Full License 


\section{Preoperative fibrinogen / albumin ratio (FAR) Can be used to predict the prognosis of urothelial carcinoma patients after radical cystectomy}

Lei Liang ${ }^{1^{*}}$, Haifeng Song ${ }^{2 *}$, Zhenhua Liu ${ }^{3 *}$, Binglei Ma ${ }^{1}$, Zhenan Zhang ${ }^{1}$, Qinhan $\mathrm{Li}^{1}$, Jun Liu ${ }^{1}$, Quan Zhang ${ }^{1}$, Shiyu Zhu ${ }^{1}$, Yisen Meng ${ }^{1}$, Qian Zhang ${ }^{1}$.

1.Department of Urology, Peking University First Hospital, Institute of Urology, Peking University, National Urological Cancer Center, Beijing 100034, China

2. Department of Urology, Beijing Tsinghua Changgung Hospital, Beijing,102200, China

3. Department of Urology, State Key Laboratory of Oncology in South China, Collaborative Innovation Center for Cancer Medicine, Sun Yat-sen University Cancer Center, Guangzhou 510060, P. R. China.

Correspondence:

Yisen Meng, Email mgyss@qq.com; telephone +8613466660291

Qian Zhang, Email zhangqianbjmu@126.com;telephone +86 01083572481

* These authors contributed equally to this work

Key Words: Bladder cancer; radical cystectomy; fibrinogen / albumin ratio (FAR); prognostic independent risk factors; survival

\section{Abstract}

Purpose: To explore the feasibility of preoperative fibrinogen / albumin ratio (FAR) as a predictor of the total and elderly patients with urothelial carcinoma after radical cystectomy.

Methods: A cohort of 275 patients who underwent radical cystectomy for bladder tumor at Peking University First Hospital from 2012 to 2016 was analyzed retrospectively. The optimal cut-off value of FAR group was determined according to the survival dependent receiver operating characteristic curve (survival-ROC). KaplanMeier curve was used to analysis the overall survival (OS), and Cox proportional 
hazards model was used for univariate and multivariate analysis.

Result: The AUC value of FAR in the survival-ROC curve is higher than other clinical indexes, and the cut-off value of FAR determined by survival-ROC is 0.0707 . We found that the median survival time $(27.63$ months vs 32.11 months, $\mathrm{P}<0.01)$ and 5 -year survival rate $(77.7 \%$ vs $92.7 \%, P<0.01)$ in the high FAR group were significantly lower than those in the low FAR group. In the multivariate survival analysis, the FAR and AJCC stages were independent risk factors for long-term prognosis. In subgroup analysis, the long-term survival of patients in AJCC I+II subgroup, G1 + G2 subgroup, G3 subgroup, lymph nodes negative subgroup and old patient subgroup were all related to FAR, while the 5-years survival of patients in AJCCIII+IV subgroup, lymph nodes positive subgroup and young patient subgroup was not related to FAR.

Conclusion: The value of FAR can be used as an independent risk factor to predict the prognosis of patients with bladder cancer after radical cystectomy, especially for patients with elder age, lymph nodes negative, or AJCC I + II stage.

\section{Introduction}

Bladder cancer is the 9th common tumor type in the world and urothelial carcinoma is the most common pathological type ${ }^{(1)}$. For patients who have muscleinvasive bladder cancer (MIBC) or high grade non muscle-invasive bladder cancer, radical cystectomy is the main treatment option ${ }^{(2)}$.However, the long-term prognosis of most patients with underwent radical cystectomy is poor because the late stage or high malignancy. ${ }^{(3)}$.Therefore, it is very meaningful to find a factor that can predict the prognosis of patients with urothelial carcinoma underwent radical cystectomy.

Most tumor patients have hypercoagulable state ${ }^{(4)}$,some studies have shown that serum high fibrinogen is related to poor prognosis of tumor patients ${ }^{(5,6)}$, and fibrinogen has also been found to play a role in many parts of tumor development ${ }^{\text {(7- }}$ 10). Albumin is considered to be an objective indicator of nutritional status ${ }^{(11,12)}$, low serum albumin level often indicates poor prognosis of tumor patients ${ }^{(13-15)}$.Previously, a retrospective study at Sun Yat sen University found that the preoperative fibrinogen 
/ albumin ratio (FAR) can be used as a predictor of postoperative prognosis in patients with soft tissue sarcoma ${ }^{(16)}$.After that, many studies were exploring the feasibility of FAR in predicting prognosis of different pathological types of tumors ${ }^{(16-19)}$.

However, there is no study to discuss the FAR value in predicting the prognosis of urothelial carcinoma patients after radical cystectomy. Here, we will explore the feasibility of FAR as a preoperative indicator to predict the prognosis of patients with urothelial carcinoma after radical cystectomy.

\section{Methods}

\section{Patients and follow-up}

Retrospective analysis the patients who underwent radical cystectomy for bladder tumor in the Peking University First Hospital from 2012 to 2016. The inclusion criteria included: 1) No neoadjuvant chemotherapy before surgery; 2) Survival more than 30 days after radical cystectomy; 3) Pathological type of bladder cancer is urothelial carcinoma; 4) Complete clinical data are maintained.

Postoperative follow-up was conducted by telephone, and the overall survival (OS)time was calculated from the surgery to October 2018 or the death of the patient. This study was approved by the Research Ethics Committee of Peking University First Hospital, Peking University.

\section{Clinical information collection}

Blood samples collected within one week before operation were used for laboratory test. The serum fibrinogen level, albumin level, leukocyte count, neutrophil count, lymphocyte count and platelet count were collected. The clinical information of patients, including gender, age, tumor pathology, BMI were collected by reviewing the medical records. The pathological stage of tumor was based on AJCC 8th Edition. FAR is defined as the ratio of serum fibrinogen $(\mathrm{mg} / \mathrm{dl})$ to albumin level $(\mathrm{g} / \mathrm{L}), \mathrm{NLR}$ as the ratio of neutrophil count to lymphocyte count, dNLR as the ratio of neutrophil count / 
(leukocyte count - neutrophil count), and PLR as the ratio of platelet count to lymphocyte count.

\section{Statistical analysis}

Chi-square $\left(\chi^{2}\right)$ test was used for data expressed as percentages between different groups. According to the survival dependent receiver operating characteristic curve (survival-ROC), the maximum Youdan index ( $\mathrm{YI}$ ) was calculated to determine the optimal cut-off value, and the area under the curve (AUCs) was calculated. The survival curve was analyzed by Kaplan-Meier method and compared by log rank test. The survival rate was analyzed by Cox proportional risk model and positive step by step. Cox proportional hazards model was used to do univariate and multivariate analyses of survival. The hazard ratios (HR) and $95 \%$ confidence interval (Cls) were reported as relative risks. A two sides $P<0.05$ was considered statistical difference. All statistical analysis was performed by R-4.0.0

\section{Results}

\section{Baseline information of patients and tumors}

The demographic variables and clinicopathological characteristics of 275 cases included in this study are listed in Table 1. A total of 231 males and 44 females, with a gender ratio of 5.25:1. The median age was 66 years (range: $35-91$ years). The median BMI was $24.00 \mathrm{~kg} / \mathrm{m}^{2}$ (range: $14.69-38.8 \mathrm{~kg} / \mathrm{m}^{2}$ ).

In terms of postoperative pathological classification, there were 135 patients (49.09\%) in AJCC stage I or stage II, 140 patients (50.91\%) in AJCC stage III or stage IV, 3 patients (1.09\%) with G1 urothelial carcinoma, 77 patients $(28.00 \%)$ with G2 urothelial carcinoma and 195 patients (70.91\%) with G3 urothelial carcinoma.

The postoperative chemotherapy plan of the patient is made according to the pathological type of the patients and the patients' wishes. 
Table1 Baseline Characteristics of Patients

\begin{tabular}{|c|c|c|}
\hline Characteristics & case & Percentage(\%) \\
\hline \multicolumn{3}{|l|}{ Sex } \\
\hline Male & 231 & 84.00 \\
\hline Female & 44 & 16.00 \\
\hline \multicolumn{3}{|l|}{ Age } \\
\hline$<60$ & 66 & 24.00 \\
\hline$\geqslant 60$ & 209 & 76.00 \\
\hline \multicolumn{3}{|l|}{ BMI } \\
\hline$<18.5$ & 9 & 3.27 \\
\hline$\geqslant 18.5$ to $<25$ & 174 & 63.27 \\
\hline$\geqslant 25.0$ & 92 & 33.45 \\
\hline \multicolumn{3}{|l|}{ AJCC stage } \\
\hline$|+| \mid$ & 135 & 49.09 \\
\hline III+IV & 140 & 50.91 \\
\hline \multicolumn{3}{|l|}{ Grade } \\
\hline G1 & 3 & 1.09 \\
\hline $\mathrm{G} 2$ & 77 & 28.00 \\
\hline G3 & 195 & 70.91 \\
\hline \multicolumn{3}{|l|}{ FAR } \\
\hline$>=0.0707$ & 193 & 70.18 \\
\hline$<0.0707$ & 82 & 29.82 \\
\hline \multicolumn{3}{|l|}{ NLR } \\
\hline$>=2.858$ & 132 & 48.00 \\
\hline$<2.858$ & 143 & 52.00 \\
\hline \multicolumn{3}{|l|}{$d N L R$} \\
\hline$>=1.730$ & 153 & 55.64 \\
\hline$<1.730$ & 122 & 44.36 \\
\hline \multicolumn{3}{|l|}{ PLR } \\
\hline$>=134$ & 160 & 58.18 \\
\hline$<134$ & 115 & 41.82 \\
\hline
\end{tabular}

\section{Optimal cut-off value of FAR}

By plotting time dependent AUCs, we found that FAR had the largest AUC compared with other clinical indicators(Figure1.a). Then, we select $t=60$ months to draw suivival-ROC.

Calculated the AUCs of different clinical index through survival-ROC(Figure1.b), the AUC of $\operatorname{FAR}(0.633)$ is the biggest,compared with $\operatorname{NLR}(A \cup C=0.536)$, $\mathrm{dNLR}(A \cup C=0.524)$ and $\operatorname{PLR}(A \cup C=0.539)$. The optimal cut-off value of FAR is 0.0707 
according to the maximum Youden index (YI). Patients with preoperative FAR higher than 0.0707 were divided into high FAR group and patients with preoperative FAR lower than 0.0707 into low FAR group.

Figure1 survival-ROC curve analyses for prognostic indicators. (a)time dependent AUCs for clinical indicators. (b) survival-ROC at time $=60$ months

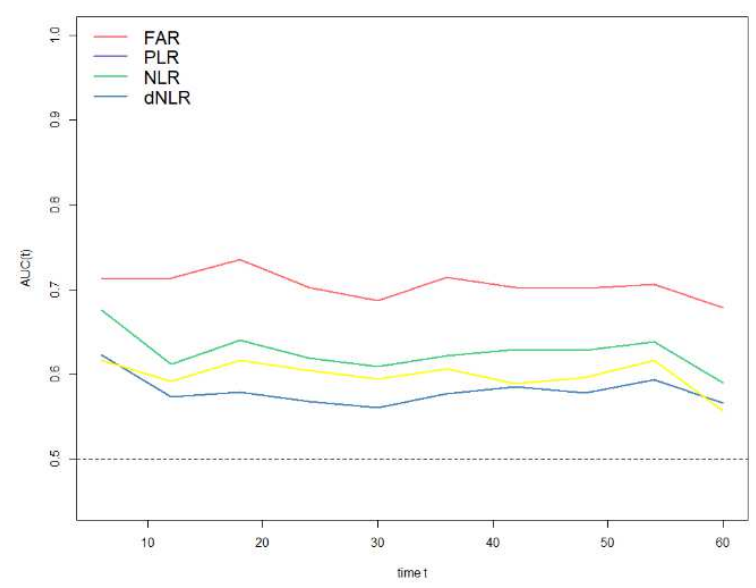

(a)

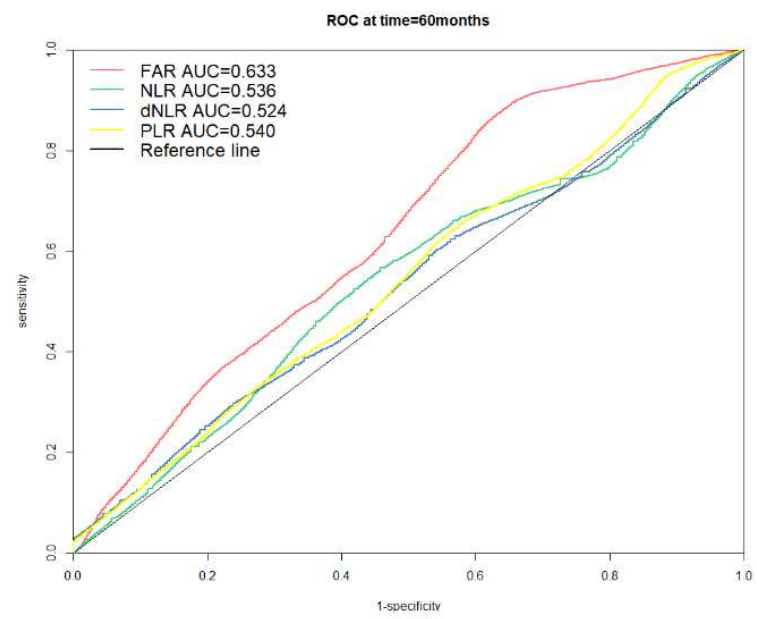

(b)

\section{Correlation of FAR with clinical information and pathological types}

$82(29.8 \%)$ patients were divided into low FAR group and $193(70.18 \%)$ patients were divided into high FAR group. There was no significant difference between the two groups in $B M I(P=0.082)$. The increased $F A R$ were relevant to patients' gender $(P=$ $0.0278)$, age $(P=0.001)$, AJCC stage $(P<0.001)$, tumor grade $(P=0.002)$ and patients' serum NLR ( $<<0.001)$, dNLR $(P<0.001)$ and PLR $(=0.010)$ (Table 2$)$. 
Table2 Correlation between the FAR and clinicopathological variables in bladder cancer patients

\begin{tabular}{|c|c|c|c|c|c|c|c|}
\hline \multirow{3}{*}{\multicolumn{2}{|c|}{ Characteristics }} & \multicolumn{5}{|c|}{ FAR } & \multirow[t]{3}{*}{$P$ value } \\
\hline & & \multicolumn{3}{|c|}{ Low FAR } & \multicolumn{2}{|c|}{ High FAR } & \\
\hline & & case & \multicolumn{2}{|c|}{ Percentage(\%) } & case & Percentage(\%) & \\
\hline Sex & & & & & & & 0.028 \\
\hline & Male & & 75 & 91.46 & 156 & 80.83 & \\
\hline & Female & & 7 & 8.54 & 37 & 19.17 & \\
\hline Age & & & & & & & 0.001 \\
\hline & $<60$ & & 30 & 36.59 & 36 & 18.65 & \\
\hline & $\geqslant 60$ & & 52 & 63.41 & 157 & 81.35 & \\
\hline BMI & & & & & & & 0.081 \\
\hline & $<18.5$ & & 0 & 0.00 & 9 & 4.66 & \\
\hline & $\geqslant 18.5$ to $<25$ & & 50 & 60.98 & 124 & 64.25 & \\
\hline & $\geqslant 25.0$ & & 32 & 39.02 & 60 & 31.09 & \\
\hline $\mathrm{AJCC} s$ & & & & & & & $<0.001$ \\
\hline & $1+11$ & & 54 & 65.85 & 81 & 41.97 & \\
\hline & III+IV & & 28 & 34.15 & 112 & 58.03 & \\
\hline Grade & & & & & & & 0.002 \\
\hline & G1 & & 3 & 3.66 & 0 & 0.00 & \\
\hline & G2 & & 30 & 36.59 & 47 & 24.35 & \\
\hline & G3 & & 49 & 59.76 & 146 & 75.65 & \\
\hline NLR & & & & & & & $<0.001$ \\
\hline & $>=2.858$ & & 24 & 29.27 & 108 & 55.96 & \\
\hline & $<2.858$ & & 58 & 70.73 & 85 & 44.04 & \\
\hline $\mathrm{dNLR}$ & & & & & & & $<0.001$ \\
\hline & $>=1.730$ & & 32 & 39.02 & 121 & 62.69 & \\
\hline & $<1.730$ & & 50 & 60.98 & 72 & 37.31 & \\
\hline PLR & & & & & & & 0.010 \\
\hline & $>=134$ & & 38 & 46.34 & 122 & 63.21 & \\
\hline & $<134$ & & 44 & 53.66 & 71 & 36.79 & \\
\hline
\end{tabular}

\section{Survival analysis}

The median follow-up time of all patients was 23.7 months. The median survival time (27.63 months vs 32.11 months, $\mathrm{P}<0.001)$ and 5 -year survival rate $(77.7 \%$ vs $92.7 \%, P<0.001)$ in the high FAR group were significantly lower than those in the low FAR group (figure 2). Multivariate Cox regression suggested that FAR could be used as an independent risk factor to predict OS in patients with radical cystectomy, and AJCC stage could also be used as an independent risk factor to predict OS in patients with 
total cystectomy. Although in the univariate analysis NLR and pathological grade show relevant to OS, but in multivariate analysis NLR and pathological grade could not be used as independent risk factors to predict OS.

Figure2. Kaplan-Meier curves showing OS according to the optimal value of FAR in bladder cancer patients

$\mathrm{FAR}+$ HighFAR + Low FAR

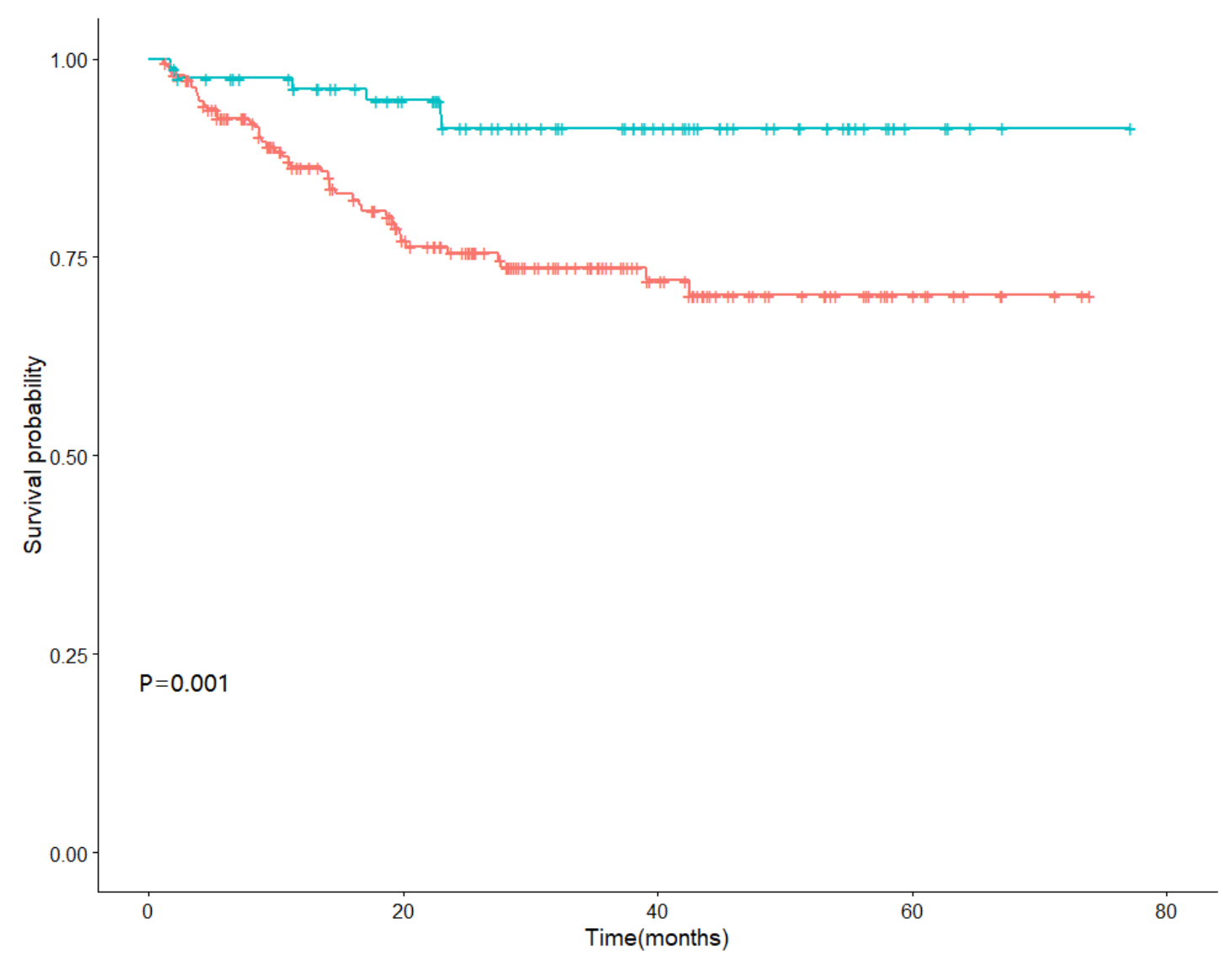

\section{Subgroup analysis}

In subgroup analysis, for patients in AJCCI+II subgroup, increased FAR predicted worse 5-year survival $(P=0.014)$, but for patients in AJCC III + IV subgroup, there was no significant difference in 5-year survival between patients with different FAR groups $(P=0.180)$ (figure3a and $3 b)$. In the subgroups of pathological grade, patients in the $G 1+G 2$ and $G 3$ subgroups showed worse 5 -year survival $(P=0.028$ and $P=0.037)$ (figure $3 c$ and $3 d$ ).In the subgroups of patients' age, patients elder than 60 years old 
assigned to the old patient subgroup,in which increased FAR predicted worse 5-year survival $(P<0.001)$, but not for patients in young patient subgroup(figure3e and $3 f$ ). For patients in lymph nodes negtive subgroup, increased FAR predicted worse 5-year survival $(P=0.001)$, but not for patients in lymph nodes positive subgroup(figure3g and 3h).

Table 3 Univariate and multivariate analyses of variables for overall survival in bladder cancer patients

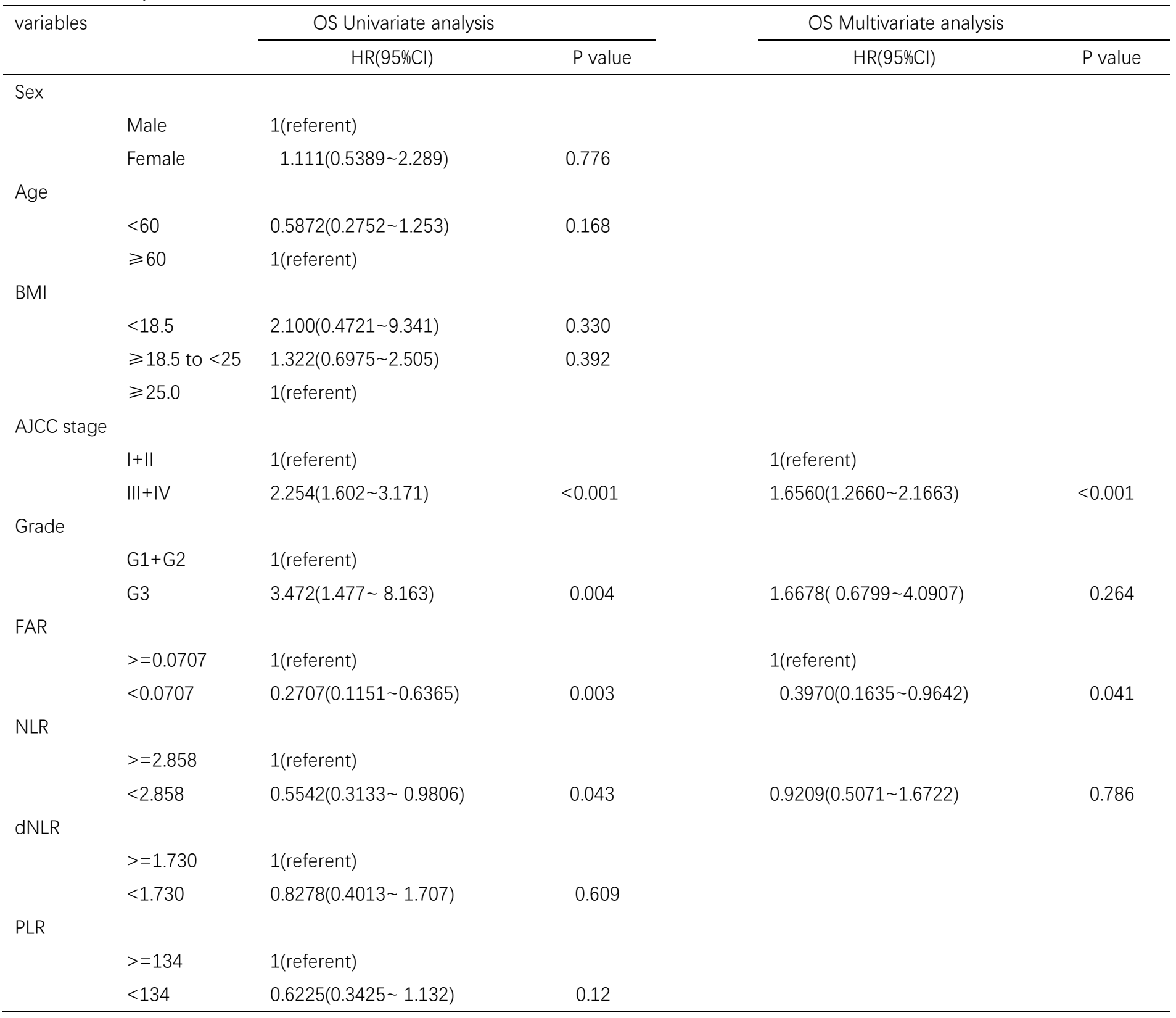


Fig. 3 Kaplan-Meier curves showing OS according to the optimal value of FAR in AJCC I+II subgroup ;(a) AJCC III+IV subgroup; (b) G1+G2 urothelial carcinoma subgroup ;(c) G3 urothelial carcinoma subgroup; (d) other pathological type subgroup; (e)old patient subgroup;(f) young patient subgroup; (g)lymph nodes positive subgroup;(h) lymph nodes negative subgroup.
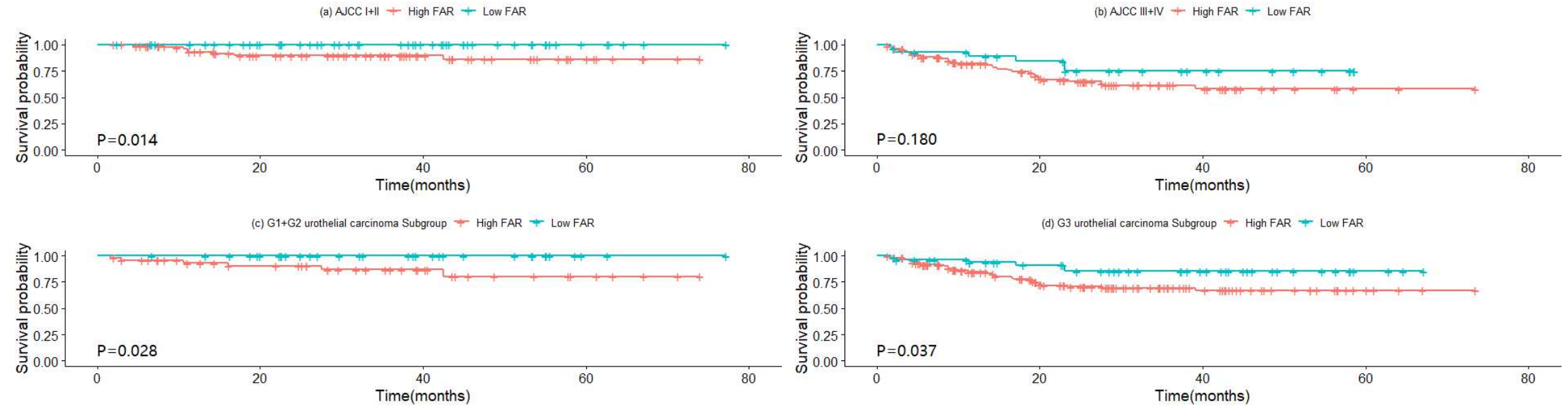

(e) old patient Subgroup + High FAR + Low FAR

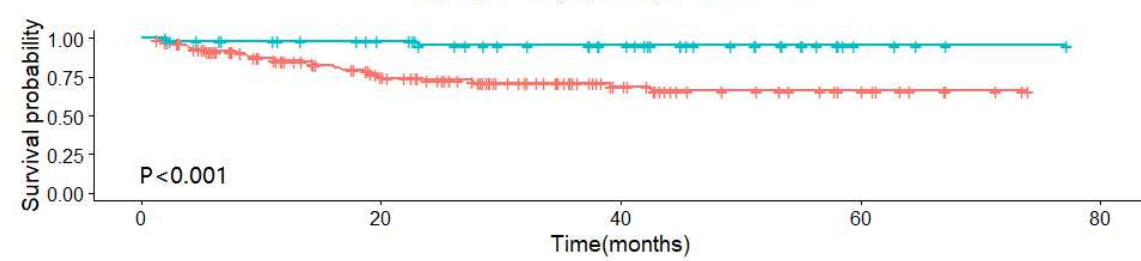

(g) lymph nodes positive Subgroup + High FAR + Low FAR

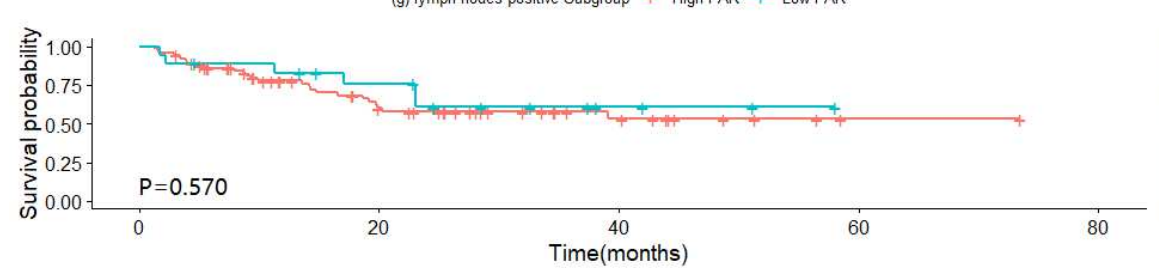

(f) young patient Subgroup - High FAR - Low FAR

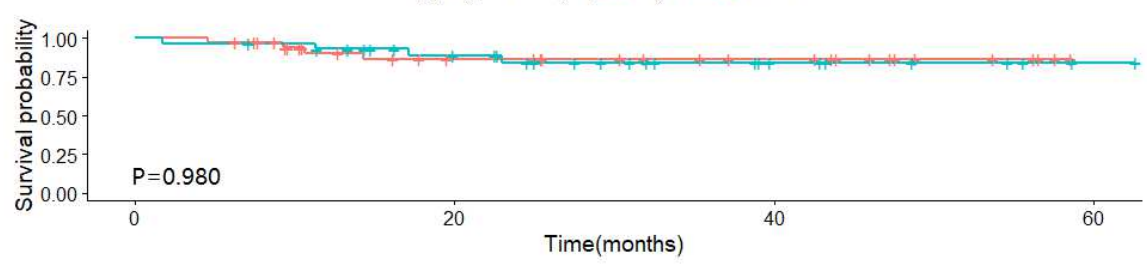

(h) lymph nodes negtive Subgroup + High FAR + Low FAR

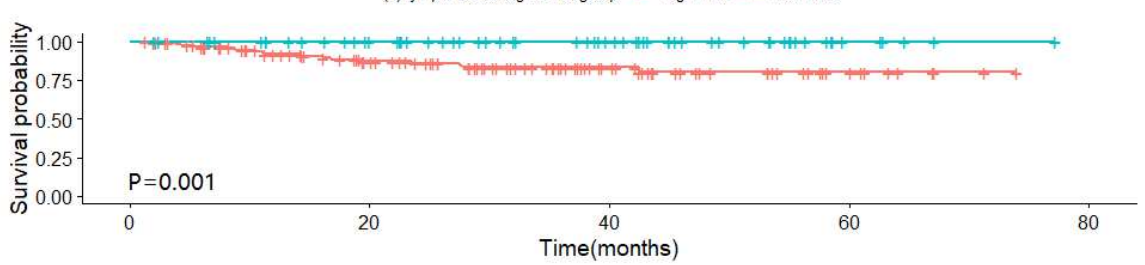


We then analyzed patients in different age subgroups. Although FAR had the highest AUCs in both subgroups, the AUC in the old patient subgroup was higher than that in the young patient subgroup (0.633 and 0.558 ,Figure 4).For old patient subgroup, in univariate analysis, AJCC stage $(P<0.001)$, FAR $(P=0.004)$, NLR $(P=0.017)$, pathological grade $(P=0.010)$ and low $B M I(P=0.039)$ were all associated with survival. In multivariate analysis, only FAR $(P=0.023)$ and AJCC stage $(P<0.001)$ associated with survival. Cox regression showed that only AJCC stage $(P<0.001)$ was associated with survival in young patient subgroup(Table 4 and Table 5).

Figure4 survival-ROC curve analyses for (a)old patient subgroup;(b) young patient subgroup.

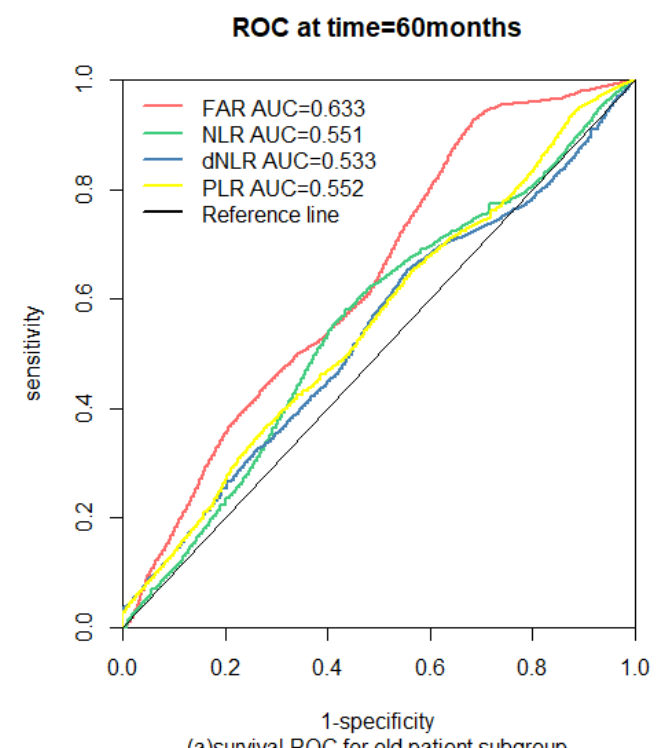

(a)survival-ROC for old patient subgroup

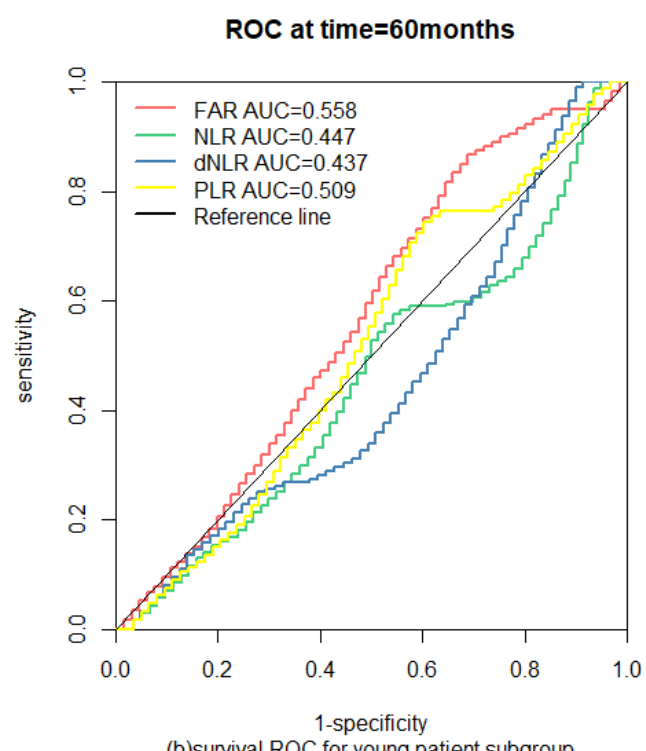

(b)survival-ROC for young patient subgroup 
Table 4 Univariate and multivariate analyses of variables for overall survival in old patient subgroup

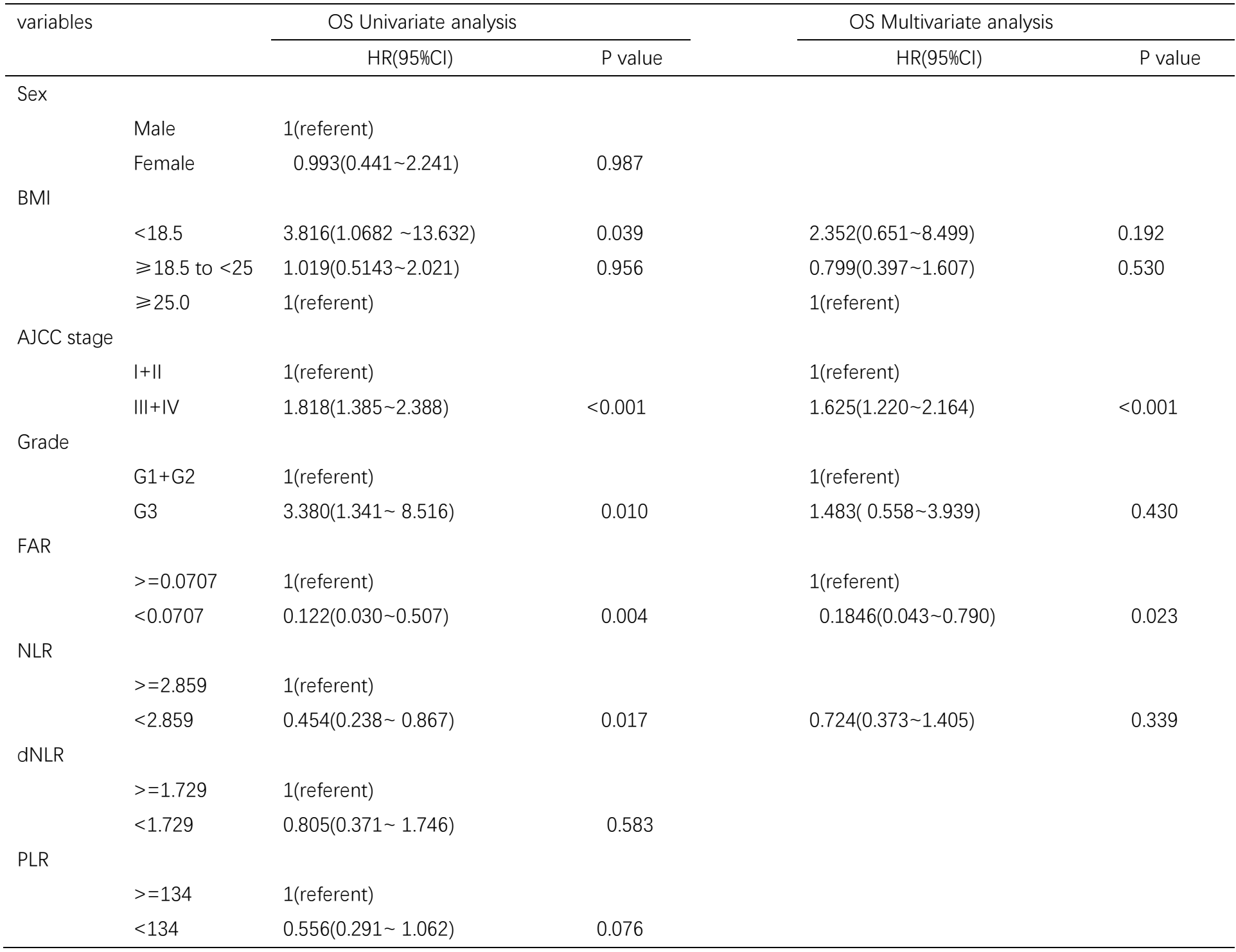


Table 5 Univariate analyses of variables for overall survival in young patient subgroup

\begin{tabular}{|c|c|c|c|}
\hline \multirow[t]{2}{*}{ variables } & \multicolumn{3}{|c|}{ OS Univariate analysis } \\
\hline & & $\mathrm{HR}(95 \% \mathrm{Cl})$ & $P$ value \\
\hline \multicolumn{4}{|l|}{ Sex } \\
\hline & Male & 1 (referent) & \\
\hline & Female & $1.757(0.354 \sim 8.712)$ & 0.490 \\
\hline \multicolumn{4}{|l|}{ BMI } \\
\hline & $<18.5$ & / & / \\
\hline & $\geqslant 18.5$ to $<25$ & $1.164(0.277 \sim 4.878)$ & 0.836 \\
\hline & $\geqslant 25.0$ & 1 (referent) & \\
\hline \multicolumn{4}{|c|}{ AJCC stage } \\
\hline & $1+\|$ & 1 (referent) & \\
\hline & III+IV & $1.818(1.385 \sim 2.388)$ & $<0.001$ \\
\hline \multicolumn{4}{|l|}{ Grade } \\
\hline & $\mathrm{G} 1+\mathrm{G} 2$ & 1 (referent) & \\
\hline & G3 & $3.477(0.448 \sim 27.17)$ & 0.235 \\
\hline \multicolumn{4}{|l|}{ FAR } \\
\hline & $>=0.0707$ & 1 (referent) & \\
\hline & $<0.0707$ & $1.020(0.254 \sim 4.090)$ & 0.978 \\
\hline \multicolumn{4}{|l|}{ NLR } \\
\hline & $>=2.858$ & 1 (referent) & \\
\hline & $<2.858$ & $1.807(0.365 \sim 8.954)$ & 0.469 \\
\hline \multicolumn{4}{|l|}{$\mathrm{dNLR}$} \\
\hline & $>=1.730$ & 1 (referent) & \\
\hline & $<1.730$ & $1.049(0.129 \sim 8.544)$ & 0.965 \\
\hline \multicolumn{4}{|l|}{ PLR } \\
\hline & $>=134$ & 1 (referent) & \\
\hline & $<134$ & $0.763(0.154 \sim 3.782)$ & 0.741 \\
\hline
\end{tabular}

\section{Discussion}

In our study, we first used the time dependent AUCs to found out FAR had higher AUC value than NLR, DNLR and PLR, which indicated FAR has better predictive efficacy compared with other clinical indicators. Then we chose time $=60$ months to draw survival-ROC curve. According to the maximum Youden index (YI) of survival-ROC, the optimal cut-off value of FAR is 0.0707 . After grouping the patients according to the FAR value, we found that the median survival time (27.63 months vs 32.11 months, $\mathrm{P}<$ $0.001)$ and 5 -years survival rate $(77.7 \%$ vs $92.7 \%, P<0.001)$ in the high FAR group were 
significantly lower than patients in the low FAR group. In the multivariate survival analysis, the FAR and AJCC stages were independent risk factors for long-term prognosis.

A meta-analysis which included 12 related studies showed that the survival rate of patients with high FAR was significantly lower than that of patients with low FAR ${ }^{(17)}$. Although the fibrinogen / albumin ratio (FAR) has been widely reported to predict tumor prognosis recently ${ }^{(16,18,19)}$. But as far as we know, this is the first article to report FAR as an independent risk factor to predict the prognosis of patients with urothelial carcinoma after radical cystectomy.

Inflammation is considered to be an important participant in the occurrence, development and metastasis of tumors. Many inflammation related indicators, including the ratio of neutrophil count to lymphocyte count (NLR) ${ }^{(20-23)}$, the ratio of neutrophil count/( leukocyte count -neutrophil count) $(d N L R)^{(20,22,24)}$ and the ratio of platelet count to lymphocyte count (PLR) ${ }^{(19-24)}$ have been reported to be used to predict the prognosis of human tumors. However, in our study, these indicators can't be used as independent risk factors to predict the prognosis of patients with urothelial carcinoma after radical cystectomy.

However, the underlying mechanism of why FAR could be used to predict tumor progression is still not completely clear. Current research shows that in tumor patients, the coagulation system is often activated abnormally, fibrinogen is an important hemostatic factor, tumor patients often show elevated serum fibrinogen level, and hyperfibrinogenemia is related to the poor prognosis of a variety of tumors $(4,7,25,26)$. Fibrinogen also plays an important role in angiogenesis, which is conducive to the development of tumors $(9,27)$. In addition, as an acute phase response protein, fibrinogen can also reflect the state of systemic inflammatory reaction, which is closely related to the development of tumor ${ }^{(26,28)}$.As an extracellular matrix protein, it can regulate the growth of tumor cells, and promote cell migration, invasion, metastasis and epithelial-mesenchymal transformation by combining with a variety of growth factors $(7,8,10,27)$. Increased fibrinogen has been shown to be associated with distant metastasis and lymph node metastasis in gastrointestinal tumors ${ }^{(29)}$. 
Albumin is considered as an objective indicator of nutritional status, and low albumin level often indicates malnutrition ${ }^{(11,12)}$. Some studies have shown that low albumin concentration can promote the inflammatory response of the body to the tumor, promote the release of a series of tumor related cytokines, thus contributing to the development of tumor ${ }^{(30)}$.In addition, in many clinical studies, low serum albumin level shows a significant correlation with the worse prognosis of different kinds of tumors ${ }^{(13-15)}$.

In our study, increased FAR was significantly related to the age of the patients ( $P$ $=0.0014)$, which may be related to the better nutritional status of the younger patients, leading to younger patients being divided into the low FAR group. The significant correlation between increased FAR and gender $(P=0.0278)$ may be caused by too few female patients (only $16 \%$ ) in our cohort.

What surprised us was that in the multivariate analysis, pathological grade could not be used as an independent risk factor to predict the prognosis of patients with urothelial carcinoma after radical cystectomy. This is contrary to our traditional acknowledge. Considering that our research object is urothelial carcinoma patients undergoing radical cystectomy, the pathological type of the majority of patients is high-grade urothelial cancer, and the majority of patients with low-grade urothelial cancer only need TUR-Bt, which results in this bias.

In subgroup analysis, the long-term survival of patients in AJCC I+II subgroup, G1 + G2 subgroup, G3 subgroup, lymph nodes negative subgroup and old patient subgroup were all related to FAR, while the 5 -years survival of patients in AJCCIII+IV subgroup, lymph nodes positive subgroup and young patient subgroup was not related to FAR. In multivariate analysis, AJCC stages can also be used as an independent risk factor to predict the prognosis of patients. AJCC stages with higher stage indicates poor prognosis, and the prediction effect of AJCC stages is stronger than far. FAR can predict the prognosis of patients with lower AJCC stages, negative lymph nodes and elder age.

We then analyzed patients in different age subgroups. FAR had the highest AUCs in both subgroups, and that in the old patient subgroup was higher than that in the young patient subgroup. In multivariate analysis, only FAR $(P=0.023)$ and AJCC stage 
$(P<0.001)$ associated with survival in old patient subgroup, and only AJCC stage $(P<$ 0.001 ) was associated with survival in young patient subgroup. This means that FAR may be a better predictor of the prognosis of elderly patients. In clinical practice, we often encounter such a scene, an elderly patient with relatively early muscle invasive bladder cancer, whether to carry out a radical operation or not needs to consider a lot of factors in clinical practice. Here, FAR as an indicator of prognosis of patient may have certain guiding significance for this problem.

Our research has several limitations. First of all, our study is a retrospective study, involving only single center and small sample, which may lead to selection bias. Second, the patient's surgery situation is not fully documented, which may lead to potential bias. Third, the different postoperative treatments were not included in our study. Therefore, our results need to be validated in a large prospective cohort study.

\section{Conclusion}

In summary, FAR value can be used as an independent preoperative risk factor to predict the prognosis of urothelial carcinoma patients after radical cystectomy, especially for patients with elder age, lymph nodes negative, or AJCC I + II stage. Large retrospective or prospective studies are still needed to verify this view.

\section{Abbreviations}

FAR

survival-ROC

OS

AUC

MIBC

$\mathrm{BMI}$

dNLR

NLR fibrinogen / albumin ratio

survival dependent receiver operating characteristic curve overall survival

area under the curve

muscle-invasive bladder cancer

body mass index

neutrophil count / (leukocyte count - neutrophil count) ratio neutrophil count/lymphocyte count ratio 


$\begin{array}{ll}\text { PLR } & \text { platelet co unt/lymphocyte count ratio } \\ \mathrm{YI} & \text { Youdan index } \\ \mathrm{HR} & \text { hazard ratio } \\ 95 \% \mathrm{Cl} & 95 \% \text { confidence interval }\end{array}$

1. We see that the, , Consent to publish, , , and subsections are missing in the section in Manuscript file. Therefore, kindly request you to please provide with statements on all of the above subsections under Declaration section in Manuscript file. In the 'Availability of data and materials' section please clearly state who should be contacted if someone wants to request the data from this study.

2. Kindly provide a clear statement on consent waiver for this study along with the full name of the IRB/ethics committee which waived the need of informed consent.

\section{Declaration}

\section{Ethics approval and informed consent}

All procedures performed in this study were in accordance with the Declaration of Helsinki (as revised in 2013). This study was approved by the Research Ethics Committee of Peking University First Hospital, Peking University. As a retrospective study and does not involve secondary injury to patients, the informed consent for the study was waived by the Research Ethics Committee of Peking University First Hospital, Peking University.

\section{Consent to publish}

Not applicable.

\section{Availability of data and materials}

All patient data are contained in the medical record of Peking University First 
Hospital. The data supporting the conclusions of this article are included within the article and its figures and tables. If you are interested in our study, please contact Lei Liang, Yisen Meng, or Qian Zhang for the data you need.

\section{Funding and competing interests}

None, and all authors declare that they have no competing interests.

\section{Authors' contribution}

Lei Liang, Haifeng Song and Zhenhua Liu wrote the main manuscript text and prepared figures and tables; Binglei Ma, Zhenan Zhang, Qinhan Li and Jun Liu collected and proofeaded the clinical data; Quan Zhang and Shiyu Zhu provided statistical and software support; Yisen Meng and Qian Zhang managed the research. All authors reviewed the manuscript.

\section{Author's information}

Lei Liang, Email: Icmllysq@126.com

Haifeng Song, Email: songhaifeng6@163.com

Zhenhua Liu, Email: liuzh9@sysucc.org.cn

Binglei Ma, Email: 343881743@qq.com

Zhenan Zhang, Email: 190392681@qq.com

Qinhan Li, Email: 1043951842@qq.com

Jun Li, Email: hmuliujun@163.com

Quan Zhang, Email: 1310301315@bjmu.edu.cn

Shiyu Zhu, Email: 18813187795@126.com

Yisen Meng, Email: mgyss@qq.com

Qian Zhang, Email: zhangqianbjmu@126.com 


\section{References}

1. Torre LA, Bray F, Siegel RL, Ferlay J, Lortet-Tieulent J, Jemal A. Global cancer statistics, 2012. CA Cancer J Clin. 2015;65(2):87-108.

2. Alfred Witjes J, Lebret T, Comperat EM, Cowan NC, De Santis M, Bruins HM, et al. Updated 2016 EAU Guidelines on Muscle-invasive and Metastatic Bladder Cancer. Eur Urol. 2017;71(3):462-75.

3. Dobruch J, Daneshmand S, Fisch M, Lotan Y, Noon AP, Resnick MJ, et al. Gender and Bladder Cancer: A Collaborative Review of Etiology, Biology, and Outcomes. Eur Urol. 2016;69(2):300-10.

4. Everett CJ, Wells BJ, Frithsen IL, Koopman RJ. Smoking, fibrinogen and cancer mortality. Journal of the national medical association. 2007;99(4):328.

5. Zhong H, Qian Y, Fang S, Wang Y, Tang Y, Gu W. Prognostic Value of Plasma Fibrinogen in Lung Cancer Patients: A Meta-Analysis. J Cancer. 2018;9(21):3904-11.

6. Li M, Wu Y, Zhang J, Huang L, Wu X, Yuan Y. Prognostic value of pretreatment plasma fibrinogen in patients with colorectal cancer: A systematic review and meta-analysis. Medicine (Baltimore). 2019;98(37):e16974.

7. Shu Y-J, Weng H, Bao R-F, Wu X-S, Ding Q, Cao Y, et al. Clinical and prognostic significance of preoperative plasma hyperfibrinogenemia in gallbladder cancer patients following surgical resection: a retrospective and in vitro study. BMC cancer. 2014;14(1):566.

8. Adams GN, Rosenfeldt L, Frederick M, Miller W, Waltz D, Kombrinck K, et al. Colon Cancer Growth and Dissemination Relies upon Thrombin, Stromal PAR-1, and Fibrinogen. Cancer Res. $2015 ; 75(19): 4235-43$.

9. Zhao C, Su Y, Zhang J, Feng Q, Qu L, Wang L, et al. Fibrinogen-derived fibrinostatin inhibits tumor growth through anti-angiogenesis. Cancer Sci. 2015;106(11):1596-606.

10. Zhang F, Wang Y, Sun P, Wang ZQ, Wang DS, Zhang DS, et al. Fibrinogen promotes malignant biological tumor behavior involving epithelial-mesenchymal transition via the $p-A K T / p-m T O R$ pathway in esophageal squamous cell carcinoma. J Cancer Res Clin Oncol. 2017;143(12):2413-24.

11. Gatta A, Verardo A, Bolognesi M. Hypoalbuminemia. Intern Emerg Med. 2012;7 Suppl 3:S193-9.

12. Garcia-Martinez R, Caraceni P, Bernardi M, Gines P, Arroyo V, Jalan R. Albumin: pathophysiologic basis of its role in the treatment of cirrhosis and its complications. Hepatology. 2013;58(5):1836-46.

13. Gupta D, Lis CG. Pretreatment serum albumin as a predictor of cancer survival: a systematic review 
of the epidemiological literature. Nutr J. 2010;9:69.

14. Konigsbrugge O, Posch F, Riedl J, Reitter EM, Zielinski C, Pabinger I, et al. Association Between Decreased Serum Albumin With Risk of Venous Thromboembolism and Mortality in Cancer Patients. Oncologist. 2016;21(2):252-7.

15. Gonzalez-Trejo S, Carrillo JF, Carmona-Herrera DD, Baz-Gutierrez P, Herrera-Goepfert R, Nunez G, et al. Baseline serum albumin and other common clinical markers are prognostic factors in colorectal carcinoma: A retrospective cohort study. Medicine (Baltimore). 2017;96(15):e6610.

16. Liang Y, Wang W, Que Y, Guan Y, Xiao W, Fang C, et al. Prognostic value of the fibrinogen/albumin ratio (FAR) in patients with operable soft tissue sarcoma. BMC Cancer. 2018;18(1):942.

17. Zhang $Y$, Xiao G. Prognostic significance of the ratio of fibrinogen and albumin in human malignancies: a meta-analysis. Cancer Manag Res. 2019;11:3381-93.

18. Zhang J, Ding Y, Wang W, Lu Y, Wang H, Wang H, et al. Combining the Fibrinogen/Albumin Ratio and Systemic Inflammation Response Index Predicts Survival in Resectable Gastric Cancer. Gastroenterol Res Pract. 2020;2020:3207345.

19. Zheng $\mathrm{Y}, \mathrm{Wu} \mathrm{C}$, Yan $\mathrm{H}$, Chen S. Prognostic value of combined preoperative fibrinogen-albumin ratio and platelet-lymphocyte ratio score in patients with breast cancer: A prognostic nomogram study. Clin Chim Acta. 2020;506:110-21.

20. Cho U, Park HS, Im SY, Yoo CY, Jung JH, Suh YJ, et al. Prognostic value of systemic inflammatory markers and development of a nomogram in breast cancer. PLoS One. 2018;13(7):e0200936.

21. Temur I, Kucukgoz Gulec U, Paydas S, Guzel AB, Sucu M, Vardar MA. Prognostic value of preoperative neutrophil/lymphocyte ratio, monocyte count, mean platelet volume, and platelet/lymphocyte ratio in endometrial cancer. Eur J Obstet Gynecol Reprod Biol. 2018;226:25-9.

22. Rajwa P, Zyczkowski M, Paradysz A, Bujak K, Bryniarski P. Evaluation of the prognostic value of LMR, PLR, NLR, and dNLR in urothelial bladder cancer patients treated with radical cystectomy. Eur Rev Med Pharmacol Sci. 2018;22(10):3027-37.

23. Kumarasamy C, Sabarimurugan S, Madurantakam RM, Lakhotiya K, Samiappan S, Baxi S, et al. Prognostic significance of blood inflammatory biomarkers NLR, PLR, and LMR in cancer-A protocol for systematic review and meta-analysis. Medicine (Baltimore). 2019;98(24):e14834.

24. Russo A, Franchina T, Ricciardi GRR, Battaglia A, Scimone A, Berenato R, et al. Baseline neutrophilia, derived neutrophil-to-lymphocyte ratio ( $d N L R)$, platelet-to-lymphocyte ratio (PLR), and outcome in non 
small cell lung cancer (NSCLC) treated with Nivolumab or Docetaxel. J Cell Physiol. 2018;233(10):633743.

25. Pichler M, Dalpiaz O, Ehrlich GC, Stojakovic T, Martin Hernandez JM, Mannweiler S, et al. Validation of the preoperative plasma fibrinogen level as a prognostic factor in a European cohort of patients with localized upper tract urothelial carcinoma. J Urol. 2014;191(4):920-5.

26. Qi Q, Geng Y, Sun M, Chen H, Wang P, Chen Z. Hyperfibrinogen is associated with the systemic inflammatory response and predicts poor prognosis in advanced pancreatic cancer. Pancreas. 2015;44(6):977-82.

27. Sahni A, Francis CW. Vascular endothelial growth factor binds to fibrinogen and fibrin and stimulates endothelial cell proliferation. Blood. 2000;96(12):3772-8.

28. Witsch E, Sela M, Yarden Y. Roles for Growth Factors in Cancer Progression. Physiology. $2010 ; 25(2): 85-101$

29. Takeuchi H, Ikeuchi S, Kitagawa Y, Shimada A, Oishi T, Isobe Y, et al. Pretreatment plasma fibrinogen level correlates with tumor progression and metastasis in patients with squamous cell carcinoma of the esophagus. J Gastroenterol Hepatol. 2007;22(12):2222-7.

30. Seaton K. Albumin concentration controls cancer. Journal of the National Medical Association. 2001;93(12):490. 

Figures

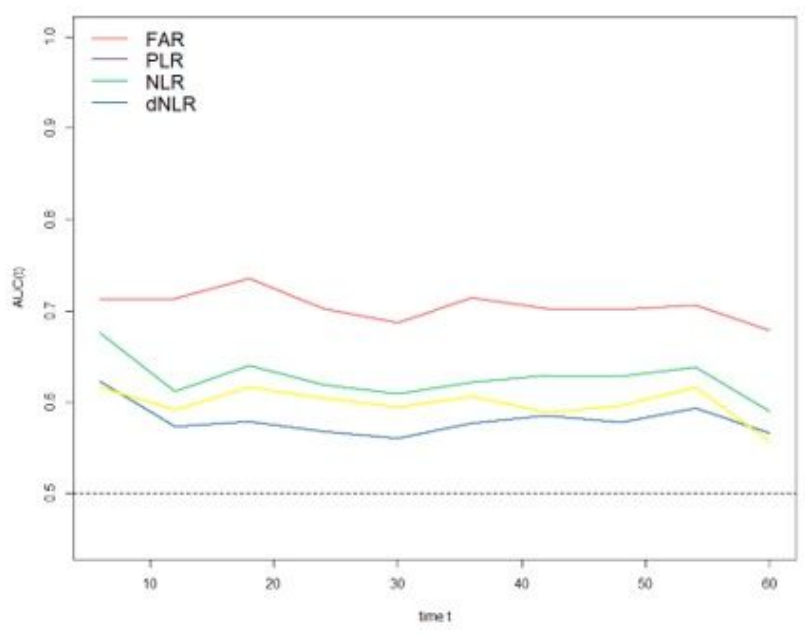

(a)

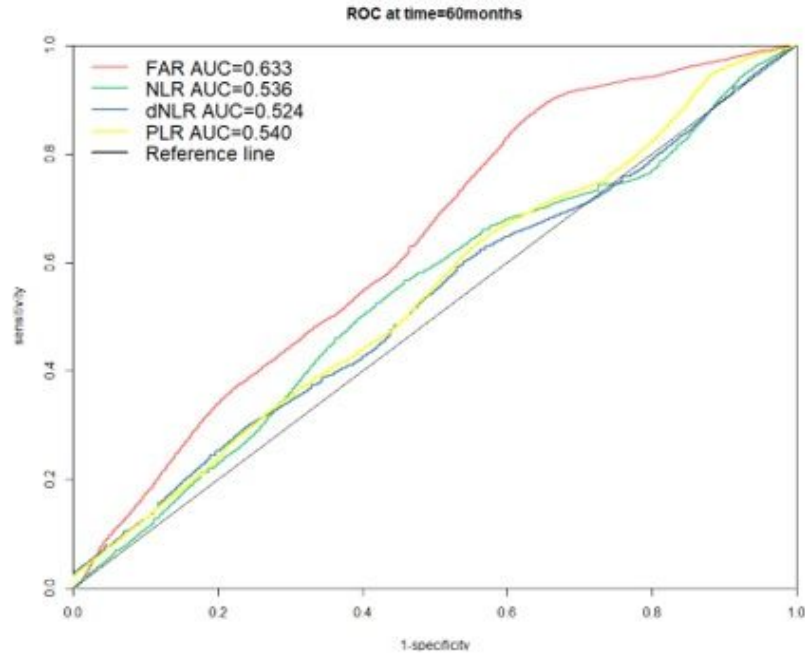

(b)

\section{Figure 1}

survival-ROC curve analyses for prognostic indicators. (a)time dependent AUCs for clinical indicators. (b) survival-ROC at time $=60$ months

FAR + High FAR + Low FAR

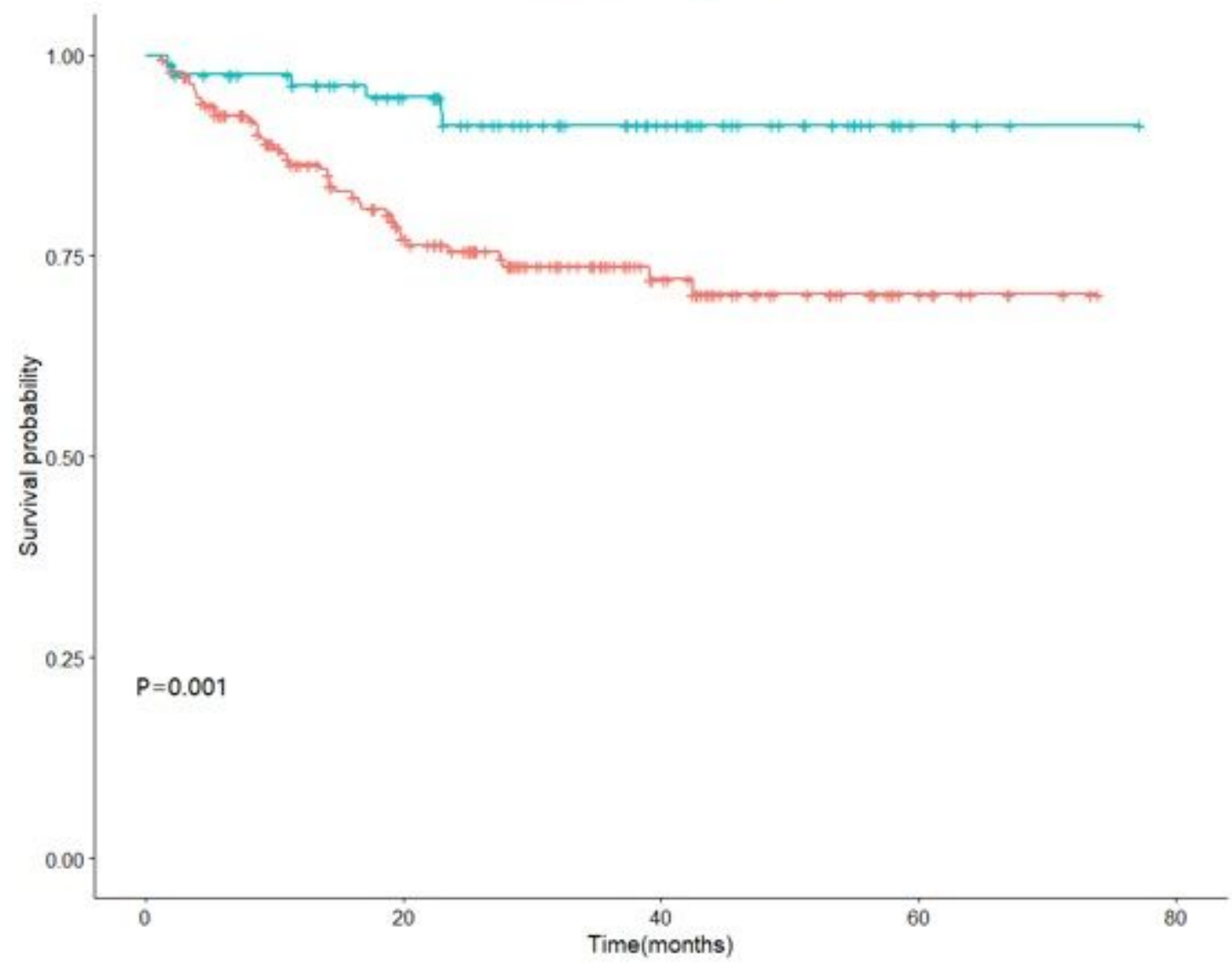


Figure 2

Kaplan-Meier curves showing OS according to the optimal value of FAR in bladder cancer patients
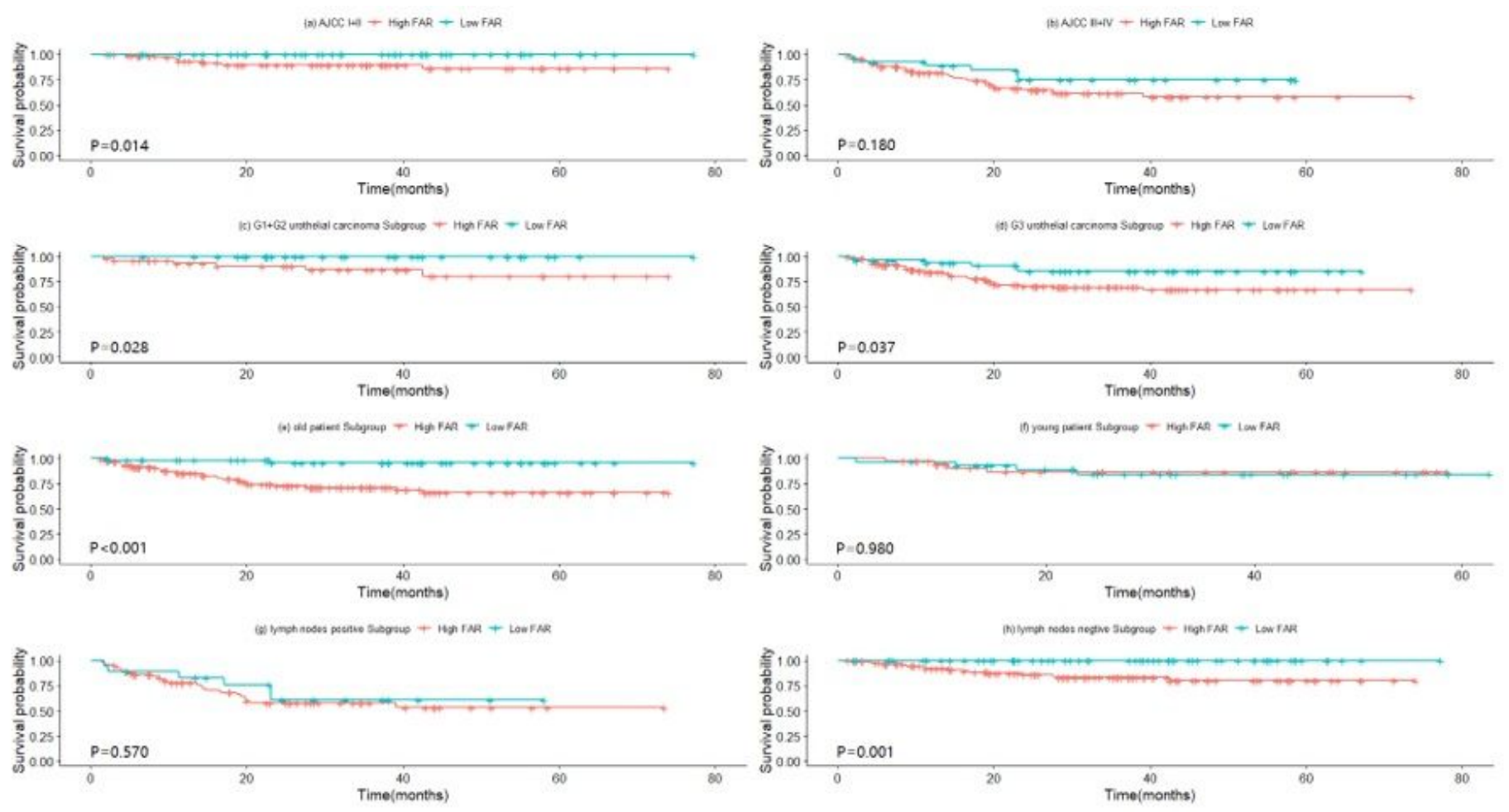

\section{Figure 3}

Kaplan-Meier curves showing OS according to the optimal value of FAR in AJCC I+II subgroup ;(a) AJCC III+IV subgroup; (b) G1+G2 urothelial carcinoma subgroup;(c) G3 urothelial carcinoma subgroup; (d)

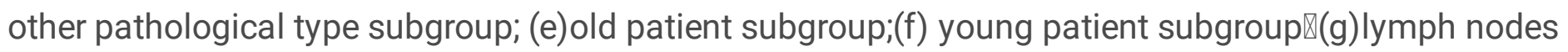
positive subgroup;(h) lymph nodes negative subgroup.

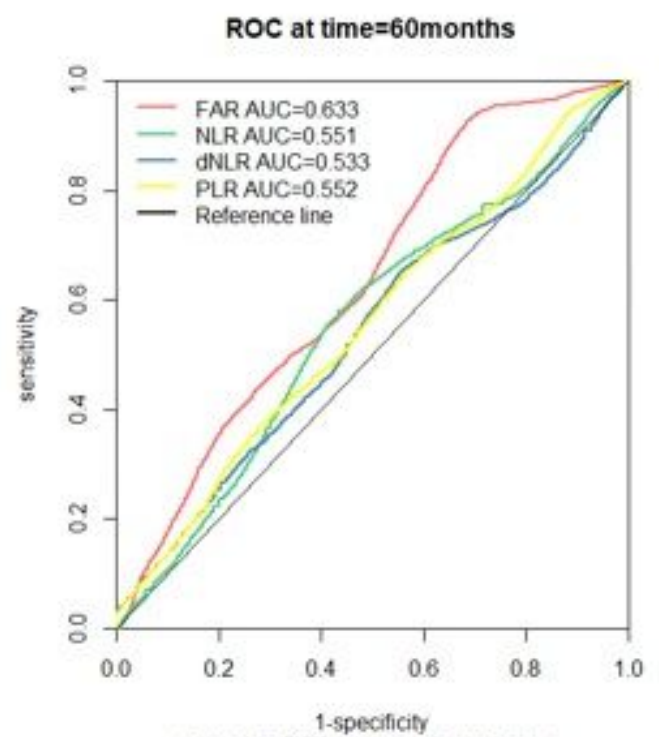

(a)survival-Roc for old patient subgrous

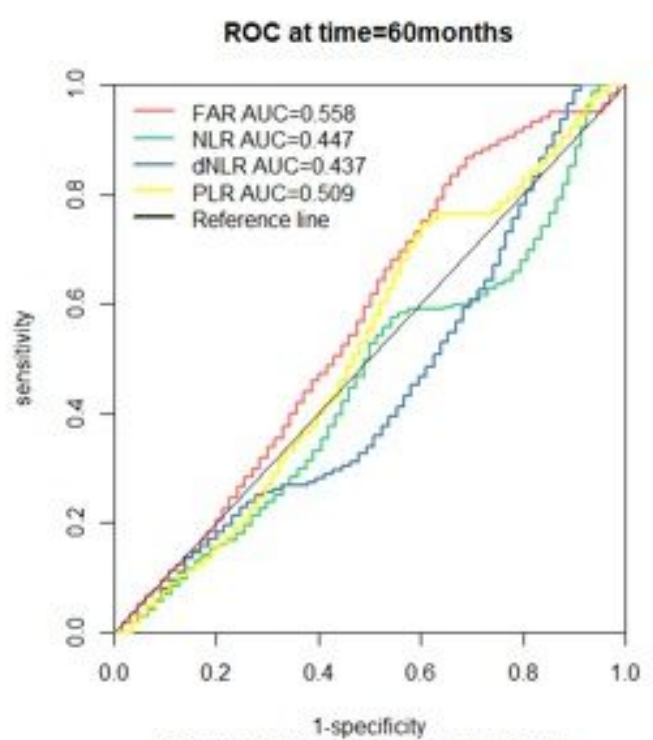

(b)survivat-RoC for young patient subgroup 
Figure 4

survival-ROC curve analyses for (a)old patient subgroup;(b) young patient subgroup. 